\title{
Note on the Polynomials which satisfy the Differential Equation
}

$$
x \frac{d^{2} y}{d x^{2}}+(\gamma-x) \frac{d y}{d x}-\alpha y=0 \text {. }
$$

By Neil M’Arthur, M.A., B.Sc.

(Read and Received 16th January 1920).

1. Laguerre has shown that if $\frac{1}{1-t} e^{\frac{x t}{1-t}}$ be expanded in ascending powers of $t$,

$$
\frac{1}{1-t} e^{\frac{x t}{1-t}}=\sum f_{n}(x) \frac{t^{n}}{n !}
$$

where $f_{n}(x)$ is a polynomial of degree $n$, satisfying the differential equation

$$
x \frac{d^{2} y}{d x^{2}}+(1+x) \frac{d y}{d x}-n y=0 .
$$

A result which differs only in the substitution of $-x$ for $x$ is given by Abel.*

Equation (1) is a special case of the equation

$$
x \frac{d^{2} y}{d x^{2}}+(\gamma-x) \frac{d y}{d x}-\alpha y=0,
$$

of which one solution is $y=F(\alpha, \gamma, x)$, where

$$
F(\alpha, \gamma, x)=1+\frac{a}{\gamma} x+\frac{\alpha(\alpha+1)}{\gamma(\gamma+1)} \frac{x^{2}}{2 !}+\frac{\alpha(\alpha+1)(\alpha+2)}{\gamma(\gamma+1)(\gamma+2)} \frac{x^{3}}{3 !}+\ldots
$$

This solution reduces to a polynomial if $\alpha$ is a negative integer, and it will be shown that the theorem of Abel and Laguerre can be extended to this polynomial.

$\S 2$. Let $y=\frac{1}{(1-t)^{p}} e^{\frac{x t}{1-t}}$,

"Laguerre, Euvres, T. I., p. 436. Abel, Euvres, T. II., p. 284. (1881). 
then

$$
\begin{aligned}
& \frac{\partial y}{\partial x}=\frac{t}{1-t} y, \frac{\partial^{2} y}{\partial x^{2}}=\frac{t}{(1-t)^{2}} y, \\
& \frac{\partial y}{\partial t}=\left[\frac{p}{(1-t)^{p+1}}+\frac{x}{(1-t)^{p+2}}\right] e^{\frac{x t}{1-t}} \\
& =\frac{p(1-t)+x}{(1-t)^{2}} y \\
& \therefore x \frac{\partial^{2} y}{\partial x^{2}}+(p+x) \frac{\partial y}{\partial x}-t \frac{\partial y}{\partial t}=0 \text {. }
\end{aligned}
$$

Hence if $f(n, p, x)$ be the coefficient of $\frac{t^{n}}{n !}$ in the expansion of $y$ in ascending powers of $t$, namely

$$
y=\Sigma f(n, p, x) \frac{t^{n}}{n !}
$$

then $f(n, p, x)$ satisfies the differential equation

$$
x \frac{d^{2} f}{d x^{2}}+(p+x) \frac{d f}{d x}-n f=0 .
$$

Comparing with equation (2) we have

$$
\begin{aligned}
f(n, p, x) & =k F^{\prime}(-n, p,-x) \\
& =k\left[1+\frac{n}{p} x+\frac{n(n-1)}{p(p+1)} \frac{x^{2}}{2 !}+\ldots+\frac{x^{n}}{p(p+1) \ldots(p+n-1)}\right]^{*}
\end{aligned}
$$

By actually expanding the two factors of $y$, as given by (3), we see that the coefficient of $x^{n}$ in $f(n, p, x)$ must be unity. Hence

$$
\boldsymbol{k}=\boldsymbol{p}(p+1) \ldots(p+n-1) \text {. }
$$

\section{§3. Recurrence Formulae.}

From (3) and (5) we have

whence we deduce

$$
\Sigma f(n, p, x) \frac{t^{n}}{n !}=(1-t) \Sigma f(n, p+1, x) \frac{t^{n}}{n !}
$$

$$
f(n, p, x)=f(n, p+1, x)-n f(n-1, p+1, x)
$$

* The result requires modification if $p$ is a negative integer. In this case the second solution of $(6)$ is required to express the coefficients for which $n>-p$. 
In like manner if

$$
\begin{aligned}
f(n, 1, x)=f_{n}=n ![1+ & n x+\frac{n(n-1)}{1^{2} \cdot 2^{2}} x^{2} \\
& \left.+\frac{n(n-1)(n-2)}{1^{2} \cdot 2^{2} \cdot 3^{2}} x^{3}+\ldots \frac{x^{n}}{n !}\right],
\end{aligned}
$$

then

whence

$$
\begin{aligned}
\Sigma f(n, p+1, x) \frac{t^{n}}{n !} & =(1-t)^{-p} \Sigma f_{n} \frac{t^{n}}{n !} \\
& =\left[1+p t+p(p+1) \frac{t^{2}}{2 !}+\ldots\right] \Sigma f_{n} \frac{t^{n}}{n !},
\end{aligned}
$$

$$
f(n, p+1, x)=f_{n}+n p f_{n-1}+\frac{n(n-1)}{1.2} p(p+1) f_{n-2}+\ldots
$$

A recurrence formula which affects $n$ only can be obtained from (4),

$$
(1-t)^{2} \frac{\partial y}{\partial t}-[p(1-t)+x] y=0 .
$$

Differentiating this $n$ times by the theorem of Leibniz and noting that

we find

$$
\left(\frac{\partial^{n} y}{\partial \iota^{n}}\right)_{t=0}=f(n, p, x) \text {, by }(5),
$$

$f(n+1, p, x)=(2 n+p+x) f(n, p, x)-n(n+p-1) f(n-1, p, x) \ldots$

\$4. Laguerre* has shown that the polynomial $f_{n}(x)$ forms the denominator of the $n^{\text {th }}$ convergent to a continued fraction for the function

$$
e^{x} \int_{x}^{\infty} \frac{e^{-x}}{x} d x
$$

It can be proved that $f(n, p+1, x)$ is similarly related to

$$
x^{p} e^{x} \int_{x}^{\infty} \frac{e^{-x}}{x^{p+1}} d x
$$

and that the numerator of the convergent whose denominator is $f(n, p+1, x)$ also satisfies a linear differential equation of the second order. The continued fraction in question has been given

* Loc. Cit., p. 428. 
by Professor Nielsen.* His method, however, does not suggest the differential equations, so that the following outline seems worth giving. The method followed is a modified form of Laguerre's.

\section{$\S 5$. Let}

$$
\begin{aligned}
S & \equiv 1-\frac{p}{x}+\frac{p(p+1)}{x^{2}}-\frac{p(p+1)(p+2)}{x^{3}}+\ldots+\frac{p(p+1) \ldots(p+2 n-1)}{x^{2 n}}, \\
& =\frac{\phi}{f}+\frac{1}{x^{2 n}} \frac{R}{f} \quad \ldots \ldots \ldots \ldots \ldots \ldots \ldots \ldots \ldots \ldots \ldots \ldots \ldots \ldots \ldots \ldots \ldots \ldots \ldots \ldots \ldots \ldots \ldots \ldots \ldots \ldots \ldots \ldots \ldots \ldots \ldots \ldots \ldots
\end{aligned}
$$

where $\phi, f, R$, are polynomials in $x$ of degree $n, n,(n-1)$, respectively. That a unique expression of the latter form exists for $S$ can be shown by the method of undetermined coefficients.

$S$ satisfies the differential equation,

$$
\frac{d S}{d x}-\frac{p+x}{x} S+\frac{a}{x^{2 n+1}}+1=0,
$$

where $a=p(p+1) \ldots(p+2 n)$.

Hence

$$
\begin{gathered}
\frac{\phi^{\prime} f-\phi f^{\prime}}{f^{2}}-\frac{2 n}{x^{2 n+1}} \frac{R}{f}+\frac{1}{x^{2 n}} \frac{R^{\prime} f-R f^{\prime}}{f^{2}} \\
-\frac{p+x}{x}\left(\frac{\phi}{f}+\frac{1}{x^{2 n}} \frac{R}{f}\right)+\frac{a}{x^{2 n+1}}+1=0 .
\end{gathered}
$$

When this identity is multiplied by $x f^{2}$, the part which is a polynomial in $x$ must vanish. Taking, as we are entitled to do, the coefficient of $x^{n}$ in $f$, and therefore in $\phi$, as unity, we find in this way,

$$
x\left(\phi^{\prime} f-\phi f^{\prime}\right)-(p+x) \phi f+x f^{2}+(a-b)=0,
$$

where $b$ is the coefficient of $x^{n-1}$ in $R$.

Writing (15) in the form

$$
\frac{\phi^{\prime} f-\phi f^{\prime}}{f^{2}}-\frac{p+x}{x} \frac{\phi}{f}=-\frac{a-b}{x f^{2}}-1,
$$

we have a linear differential equation of the first order in $\frac{\phi}{f}$ whose solution, as given by the usual rule, is

$$
\frac{e^{-x}}{x^{p}} \frac{\phi}{f}=\int_{x}^{\infty} \frac{e^{-x}}{x^{p}} d x+(a b) \int_{x}^{\infty} \frac{e^{-x}}{x^{p+1} f^{2}} d x \text {. }
$$

\footnotetext{
* Theorie des Integrallogarithmus. Leipzig (1906); p. 45,
} 
$\frac{\phi}{f}$ will therefore be the $n^{\text {th }}$ convergent to a continued fraction for

$$
x^{p} e^{x} \int_{x}^{\infty} \frac{e^{-x}}{x^{p}} d x
$$

if we can prove that

$$
r_{n}=\operatorname{Lt}_{n \rightarrow \infty}(a-b) \int_{x}^{\infty} \frac{e^{-x}}{x^{p+1} f^{2}} d x=0 .
$$

§6. The differential equations for $f$ and $\phi$.

The form of equation (15) shows that

(i) $f$ has no repeated zero, since a common factor of $f$ and $f^{\prime}$ would be a factor of $(a-b)$.

(ii) $\phi$ and $f$ have no common factor.

Differentiating (15) to get rid of the unknown term $b$, we have

or

$$
\begin{gathered}
x\left(\phi^{\prime \prime} f-\phi f^{\prime \prime}\right)+\left(\phi^{\prime} f-\phi f^{\prime}\right)-(p+x)\left(\phi^{\prime} f+\phi f^{\prime}\right) \\
-\phi f+2 x f f^{\prime}+f^{2}=0,
\end{gathered}
$$

$\left[x \phi^{\prime \prime}+(1-p-x) \phi^{\prime}-\phi+f+2 x f^{\prime}\right] f=\left[x f^{\prime \prime}+(1+p+x) f^{\prime}\right] \phi$.

By virtue of (i) and (ii) this identity can only be true if

$$
\begin{gathered}
x f^{\prime \prime}+(1+p+x) f^{\prime}=k f \\
x \phi^{\prime \prime}+(1-p-x) \phi^{\prime}-\phi+f+2 x f^{\prime}=k \phi
\end{gathered}
$$

where $k$ is a constant. Since the coefficient of $x^{n}$ in $f$ and $\phi$ is unity, we obtain $k=n$.

Hence the differential equation for $f$ is

$$
x f^{\prime \prime}+(1+p+x) f^{\prime}-n f=0
$$

whence $f=f(n, p+1, x)$, as defined by (7).

The differential equation for $\phi$ is then

$$
x \phi^{\prime \prime}+(1-p-x) \phi^{\prime}-(n+1) \phi+f+2 x f^{\prime}=0
$$

where $f$ has the above value.

\section{\$7. Convergency.}

To establish (17) we require the value of $b$. This is obtained by equating the coefficients of $x^{n-1}$ in

$$
x^{2 n} \cdot S \cdot f(n, p+1, x)=x^{2 n} \phi+R .
$$


We find, using (14),

$$
\begin{aligned}
a-b & =\frac{n !(p+n) !}{(p-1) !} \\
r_{n} & =\frac{n !(p+n) !}{(p-1) !} \int_{x}^{\infty} \frac{e^{-x}}{x^{p+1} f^{2}} d x .
\end{aligned}
$$

From (7) it is evident that $f^{\prime}$ is positive for positive values of $x$, so that $f$ is an increasing function of $x$ for $x>0$.

Hence

$$
\begin{aligned}
0<r_{n} & <\frac{n !(p+n) !}{p !} \cdot \frac{1}{x^{p+1} f^{2}} \int_{x}^{\infty} e^{-\alpha} d x, \\
& <\frac{n !(p+n) !}{p ![(p+1) \ldots(p+n)]^{2}} \frac{e^{-x}}{x^{p+1}},
\end{aligned}
$$

where $f(x)$ has been replaced by $f(0)$.

$$
\text { i.e. } \begin{aligned}
0<r_{n} & <\frac{n !}{(p+1) \ldots(p+n)} \frac{e^{-x}}{x^{p+1}}, \\
& <\frac{p !}{(n+1) \ldots(n+p)} \frac{e^{-x}}{x^{p+1}} .
\end{aligned}
$$

and therefore $\underset{n \rightarrow \infty}{\operatorname{Lt}} r_{n}=0$.

\$. The recurrence formulae for $f$ and $\phi$.

We have seen, (12), that

$$
f_{n+1}=(2 n+p+1+x) f_{n}-n(n+p) f_{n-1},
$$

when $f_{n} \equiv f(n, p+1, x)$.

It can be shown that

$$
\phi_{n+1}=(2 n+p+1+x) \phi_{n}-n(n+p) \phi_{n-1} .
$$

As the proof is somewhat lengthy, in the form in which $I$ have obtained it, it is not given here.

These relations enable us to form the continued fraction whose $n^{\text {th }}$ convergent is $\frac{\phi_{n}}{f_{n}}$. We find

$$
\int_{x}^{\infty} \frac{e^{-x}}{x^{p}} d x=\frac{e^{-x}}{x^{p}}\left[1-\frac{p}{x+p+1-} \frac{p+1}{x+p+3-} \frac{2(p+2)}{x+p+5-\cdots]}\right.
$$


But

$$
\int_{x}^{\infty} \frac{e^{-x}}{x^{p}} d x=\frac{e^{-x}}{x^{p}}-p \int_{x}^{\infty} \frac{e^{-x}}{x^{p+1}} d x
$$

\section{Hence}

$$
\int^{\infty} \frac{e^{-x}}{x^{p+1}} d x=\frac{e^{-z}}{x^{p}}\left[\frac{1}{x+p+1-} \frac{p+1}{x+p+3-} \frac{2(p+2)}{x+p+5-} \ldots\right]
$$

which is Nielsen's result.

If the $n^{\text {th }}$ convergent to the second continued fraction be $\frac{g_{n-1}}{f_{n}}$, the differential equation for $g_{n-1}$ is

$$
x \frac{d^{2} g}{d x^{2}}+(1-p-x) \frac{d g}{d x}-(n+1) g+2 f^{\prime}=0,
$$

which is rather simpler than the corresponding equation for $\phi$. 